\title{
Correlation Functions of Multisite Interaction Spin-S models on the Bethe-like Lattices
}

\author{
R.G. Ghulghazaryan \\ Department of Theoretical Physics, Yerevan Physics Institute, \\ Alikhanian Br.2, 375036 Yerevan, Armenia
}

October 11, 2018

\begin{abstract}
Multisite interaction spin-S models in an external magnetic field are studied recursively on the Bethe-like lattices. The transfer-matrix method is extended to calculate exactly the two-spin correlation functions. The exact expressions for the correlation length and magnetic susceptibility are derived for spin- $1 / 2$ models. The singularity of the correlation length with critical index $\nu=1$ and the proportionality of magnetic susceptibility to correlation length in the second order phase transition region of spin- $1 / 2$ ferromagnetic models on the Bethe-like lattices are established analytically.
\end{abstract}

\section{Introduction}

The investigation of physical systems such as binary alloys [1], classical fluids [2], liquid bilayers [4], solid ${ }^{3} \mathrm{He}$ [3], rare gases [5] and anisotropic magnets such as $\mathrm{CeBi}$, EuSe, etc. lead to multisite interaction (MSI) spin and Heisenberg models. This models have a complex phase diagrams and one of the challenging problems in modern statistical mechanics is the effect of multisite interactions on the properties of order-disorder and order-order phase transitions. Mean-field theory which is most used and the simplest method for obtaining approximate results, has been shown to give poor results for such systems [3, 6, 7]. Therefore, to obtain better approximations high temperature series expansions [3], Monte Carlo simulations [7], Bethe lattice and Cayley tree approximations [8]-[10] are used. Although, there are lots of approximate methods one need to have simple but accurate technique to approximate multisite interaction (MSI) spin systems. After the pioneer works of Bethe and Peierls [11] the Bethe lattice approximation has attracted particular interest because the calculations on the Bethe lattice are rather simple and results reflects essential features of the systems, even when conventional mean-field theories fail [12]. The main difference between the Cayley tree and Bethe lattice as discussed in Ref. [13] is that in Cayley tree the surface plays a very important role. The number of sites on it's surface is a finite fraction of the total sites even in the thermodynamic limit. As a consequence, the models on the Cayley tree exhibit quite unusual critical behavior without long-range order [9]-[17]. To overcome the problems with boundary one considers only 
properties of sites deep within the interior of the Cayley tree. The union of such equivalent sites is regarded as forming Bethe lattice and it is assumed to have a translational symmetry like any regular lattice.

It was shown by Monroe [8] that one can obtain much better approximations than on the Bethe lattice by using Husimi or more general Bethe-like lattices to investigate MSI systems, especially in case of frustration. By going to Bethe-like lattice one constructs the system from different type of blocks and, hence, can differentiate between two lattices having the same coordination numbers, such as triangle and simple cubic lattices, whereas in the case of Bethe lattice it is impossible. Furthermore, the various options regarding the connection of the basic building blocks, the symmetry of Hamiltonian and, as a consequence, the possibility of division of such lattices on shells are of importance (see Sect. 2).

In the investigation of critical phenomena the correlation functions play an important role. It is established that near second order phase transition critical temperature $T_{c}$ the fluctuations in system rise and grappled all the system at $T_{c}$. It is believed that singularities of thermodynamic quantities such as the specific heat and the susceptibility at $T_{c}$ are related to the increase of fluctuations in the system or, in other words, to divergence of correlation length at $T_{c}$ 18. Since two-spin correlation functions can be measured experimentally as linear response to an adiabatic or isotermic applied field, or scattering of neutrons or electromagnetic waves [19]- [20] it is desirable to have an analytic form of the correlation function to locate phase transitions and a deeper understanding of phase transition phenomena.

Recently spin-spin correlation functions of spin- $S$ models in an external magnetic field was calculated on the Bethe lattice [21], where the crucial role of Bethe lattice dimensionality was demonstrated in determining the critical behavior of correlation length. It was shown that correlation length diverges at the critical point of $m$ and $\chi$ for the Bethe lattice Ising model with critical exponents $\nu=1$, which is different from the mean-field critical exponent $\nu=\frac{1}{2}$.

In this paper we apply the transfer matrix method to analytical calculation of the twospin correlation functions of the multisite interaction spin- $S$ models in the presence of an external magnetic field on the general Bethe-like lattices with Hamiltonian symmetric under the permutation of spins at any $p$-polygon of the lattice. The derivation of the analytic expression for correlation functions for arbitrary spin- $S$ is presented in Sect. 2. For spin- $\frac{1}{2}$ case, taking into account the dimensionality of the Bethe-like lattice, the exact expressions of the correlation length $\xi$ and magnetic susceptibility $\chi$ are derived in Sect. 3. It is demonstrated exactly that at $T=T_{c}$ the correlation length $\xi$ diverges with critical exponent $\nu=1$ and $\chi \sim \xi$ for arbitrary $p$-polygon Bethe-like lattice with $q$-polygons going out from each site.

\section{The Model}

The Bethe-like lattice may be constructed as follows : Connecting $(q-1) p$-polygons at a single base site one gets a first generation branch (Fig.1.(a)). Connecting $(q-1)$ first generation branches to each site of a new p-polygon (basic block), except the base site of this system one gets the second generation branch (Fig. 1.(b)). Continuing this process one gets higher generation branches. To complete the system, one connects $q$ nth generation branches at their

\footnotetext{
${ }^{1} p$-polygon is a closed polygon with $p$ edges (sites), for example 2-polygon is a line, 3-polygon is a triangle etc.
} 
base site (0th or central site) (Fig. 1.(c)). The set of sites deep within this system is called the Bethe-like lattice (see the Introduction). As we see only topological and connectivity properties are used to define the Bethe-like lattice. This system is not a lattice in a conventional sense.

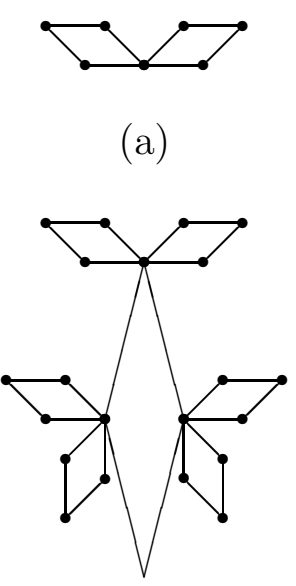

(b)

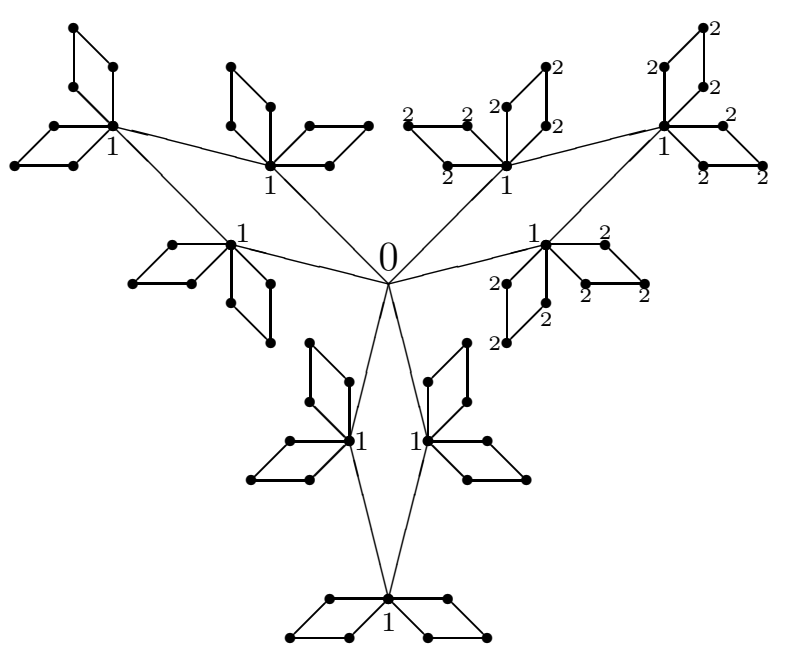

(c)

Figure 1: The recursive structure of 4-polygon Bethe-like lattice with $q=3$. (a) The first generation branch; (b) the second generation branch; (c) the central part of 4-polygon Bethelike lattice with $q=3$, the numbers $0,1,2$ indicate shells.

The Bethe and Bethe-like lattices are usually viewed as ramified trees embedded in infinite dimensional Euclidean space, with a constant vertex connectivity. The fact that Bethe and Bethe-like lattices usually are viewed as "infinite-dimensional" follows from the definition of lattice dimensionality $d$ in Euclidean space given in Ref. [13]

$$
d=\lim _{n \rightarrow \infty} \frac{\ln \tilde{C}_{n}}{\ln n}
$$

where $\tilde{C}_{n}$ is the total number of sites within $n$ steps of a given site. For a Bethe-like lattice

$$
\tilde{C}_{n}=1+\sum_{m=1}^{n} C_{m}, \quad C_{n}=q(p-1)[(q-1)(p-1)]^{n-1}, \quad C_{0}=1,
$$

where $C_{n}$ is the number of sites on the nth shell. The substitution of this expression into (1) gives $d=\infty$, so in this sense the Bethe-like lattice is "infinite-dimensional".

Recently it was shown that Bethe-like lattices can be embedded in two-dimensional space of constant negative curvature (the $\mathrm{H} 2$ hyperbolic plane) with fixed bond angles and lengths. This hierarchical structures like Bethe-like lattices can be described as a conformal tiling of the hyperbolic plane and their metric properties are studied [22].

After this brief survey of the geometrical properties of Bethe-like lattices let us now define the Hamiltonian of the multisite interaction (MSI) system. The general MSI spin Hamiltonian 
have the form

$$
\mathcal{H}=-\frac{J_{p}}{S^{p}} \sum_{\langle\text {polygons }\rangle} \prod S_{j}-\frac{h}{S} \sum_{i} S_{i},
$$

where $S_{i}$ takes values $S, S-1, \ldots,-S+1,-S$; the first sum goes over all the $p$-polygons of the lattice and $\Pi$ means the product of all spins placed on a $p$-polygon; the second term presents the interaction with an external magnetic field and the sum goes over all the sites of the lattice.

The advantage of the Bethe-like lattice is that for the models formulated on it, the exact recurrence relation can be derived and the dynamical systems theory may be used to explore the thermodynamic properties of the models [9, 10, 23]. Let us denote the partition function of the $N-t h$ generation branch with the basic spin in state $S$ as $B(N, S)$. By cutting the lattice at the basic $p$-polygon one will obtain $(q-1)(p-1)$ interacting $(N-1)-t h$ generation branches

$$
B\left(N, S_{0}\right)=\sum_{S_{1}, \ldots, S_{p-1}} w\left(S_{0}, S_{1}, \ldots, S_{p-1}\right) B^{\gamma}\left(N-1, S_{1}\right) \cdots B^{\gamma}\left(N-1, S_{p-1}\right),
$$

where $S_{0}, S_{1}, \ldots, S_{p-1}$ are the spins at the basic $p$-polygon, $w\left(S_{0}, S_{1}, \ldots, S_{p-1}\right)$ is the statistical weight of the basic p-polygon and $\gamma=q-1$.

$$
w\left(S_{0}, S_{1}, \ldots, S_{p-1}\right)=\exp \left\{\frac{\beta J_{p}}{S^{p}} S_{0} S_{1} \cdots S_{p-1}+\frac{\beta h}{q S}\left(S_{0}+\cdots+S_{p-1}\right)\right\}
$$

where $\beta=1 / k T$. By cutting apart the lattice at the central site one can obtain for the partition function $\mathcal{Z}=\sum e^{-\beta \mathcal{H}}$ the following expression

$$
\mathcal{Z}_{N}=\sum_{S_{0}} B^{q}\left(N, S_{0}\right)
$$

From Eq. (5) follows that $w\left(S_{0}, S_{1}, \ldots, S_{p-1}\right)$ is a symmetric function under the permutation of its arguments $S_{0}, S_{1}, \ldots, S_{p-1}$. Now it is easy to check that all the spins at the $n-t h$ generation branch surface are equivalent and we refer to them as $n-t h$ shell (see Fig. 1.(c)).

Let us now define the auxiliary quantities $x(s)$ as follows

$$
x_{N}(s)=\frac{B(N, s)}{B(N, S)}, \quad x_{N}(S)=1 \quad \text { and } \quad s=S-1, \ldots,-S .
$$

The thermodynamic quantities such as the magnetization, the specific heat etc. and even thermodynamic potentials such as the free energy 24 may be expressed in terms of $x(s)$. Of course, $x(s)$ has no direct physical meaning, but $\{x(s)\}$ determine the state of the system in the thermodynamic limit. From Eqs. (田) and (7) we can get $2 S$ recurrence equations for $\{x(s)\}$

$$
x_{N+1}(s)=\frac{\sum_{S_{1}, \ldots, S_{p-1}} w\left(s, S_{1}, \ldots, S_{p-1}\right) x_{N}^{\gamma}\left(S_{1}\right) \cdots x_{N}^{\gamma}\left(S_{p-1}\right)}{\sum_{S_{1}, \ldots, S_{p-1}} w\left(S, S_{1}, \ldots, S_{p-1}\right) x_{N}^{\gamma}\left(S_{1}\right) \cdots x_{N}^{\gamma}\left(S_{p-1}\right)} .
$$

Using Eqs. (6) and (4) one can express the magnetization of the central site as follows

$$
m_{\mu}=\left\langle\left(\frac{S_{0}}{S}\right)^{\mu}\right\rangle=\frac{1}{S^{\mu}} \frac{\sum_{S_{0}} S_{0}^{\mu} x_{N}^{q}\left(S_{0}\right)}{\sum_{S_{0}} x_{N}^{q}\left(S_{0}\right)}
$$


where $\langle\ldots\rangle$ denotes the thermal average and $\mu$ takes values from 1 to $2 S$.

Further we will restrict our treatment to the case when the series of solutions of recurrence equations (8) converges to a stable fixed point as $N \rightarrow \infty$. Note that for stable fixed point of Eq. (\$) the difference between $x_{N}(s)$ and $x_{N-i}(s)$ disappears for $N \rightarrow \infty$

$$
\lim _{N \rightarrow \infty} x_{N-i}(s)=x(s)
$$

for all finite values $i$. The corresponding fixed points equations of the mapping (\$) have the form

$$
\sum_{S_{i}}\left[w\left(s, S_{1}, \ldots, S_{p-1}\right)-x(s) w\left(S, S_{1}, \ldots, S_{p-1}\right)\right] x^{\gamma}\left(S_{1}\right) \cdots x^{\gamma}\left(S_{p-1}\right)=0,
$$

where $i=1, \ldots, p-1$.

The correlation functions are defined as follows

$$
g(n)=\frac{1}{S^{2}}\left(\left\langle S_{0} S_{n}\right\rangle-\left\langle S_{0}\right\rangle\left\langle S_{n}\right\rangle\right)
$$

where spin $S_{0}$ belongs to the central polygon and $S_{n}$ located somewhere in the $n t h$ shell. Using denotation (7) and recurrence relations (田) we can express $\left\langle S_{0} S_{n}\right\rangle$ as follows

$$
\begin{aligned}
\left\langle S_{0} S_{n}\right\rangle & =\lim _{N \rightarrow \infty} \frac{\sum S_{0} x_{N}^{\gamma}\left(S_{0}\right) w\left(S_{0}, y_{1}, \ldots, t_{1}, z_{1}\right) x_{N-1}^{\gamma}\left(y_{1}\right) \cdots x_{N-1}^{\gamma}\left(t_{1}\right) \times}{\sum x_{N}^{\gamma}\left(S_{1}\right) w\left(S_{1}, y_{1}, \ldots, t_{1}, z_{1}\right) x_{N-1}^{\gamma}\left(y_{1}\right) \cdots x_{N-1}^{\gamma}\left(t_{1}\right) \times} \\
& \frac{x_{N-1}^{\gamma-1}\left(z_{1}\right) w\left(z_{1}, y_{2}, \ldots, t_{2}, z_{2}\right) x_{N-2}^{\gamma}\left(y_{2}\right) \cdots x_{N-2}^{\gamma}\left(t_{2}\right) \times \cdots}{x_{N-1}^{\gamma-1}\left(z_{1}\right) w\left(z_{1}, y_{2}, \ldots, t_{2}, z_{2}\right) x_{N-2}^{\gamma}\left(y_{2}\right) \cdots x_{N-2}^{\gamma}\left(t_{2}\right) \times \cdots} \\
& \frac{x_{N-n+1}^{\gamma-1}\left(z_{n-1}\right) w\left(z_{n-1}, y_{n}, \ldots, t_{n}, S_{n}\right) x_{N-n}^{\gamma}\left(y_{n}\right) \cdots x_{N-n}^{\gamma}\left(t_{n}\right) x_{N-n}^{\gamma}\left(S_{n}\right) S_{n}}{x_{N-n+1}^{\gamma-1}\left(z_{n-1}\right) w\left(z_{n-1}, y_{n}, \ldots, t_{n}, S_{n}\right) x_{N-n}^{\gamma}\left(y_{n}\right) \cdots x_{N-n}^{\gamma}\left(t_{n}\right) x_{N-n}^{\gamma}\left(S_{n}\right)}
\end{aligned}
$$

where sums run over all spin states displayed.

To use the transfer matrix method let us introduce the following symmetric matrixes with elements

$$
\begin{aligned}
& M_{i}\left(z_{i}, z_{i+1}\right)=\left[x_{N-i}\left(z_{i}\right) x_{N-i-1}\left(z_{i+1}\right)\right]^{\frac{\gamma-1}{2}} \times \\
& \sum_{y_{i+1}, \ldots, t_{i+1}} w\left(z_{i}, y_{i+1}, \ldots, t_{i+1}, z_{i+1}\right) x_{N-i-1}^{\gamma}\left(y_{i+1}\right) \cdots x_{N-i-1}^{\gamma}\left(t_{i+1}\right), \\
& A\left(S_{n}, S_{0}\right)=\left[x_{N-n}\left(S_{n}\right) x_{N}\left(S_{0}\right)\right]^{\frac{\gamma+1}{2}}, \\
& A^{\prime}\left(S_{n}, S_{0}\right)=S_{n} S_{0}\left[x_{N-n}\left(S_{n}\right) x_{N}\left(S_{0}\right)\right]^{\frac{\gamma+1}{2}} \text {. }
\end{aligned}
$$

For stable fixed points $\{x(s)\}$ of Eq. (8) all matrixes $M_{i}$ coincide, $M_{i} \equiv M$ for all $i$. Thus, we can rewrite $\left\langle S_{0} S_{n}\right\rangle$ as follows

$$
\left\langle S_{0} S_{n}\right\rangle=\frac{\operatorname{Tr}\left(A^{\prime} M^{n}\right)}{\operatorname{Tr}\left(A M^{n}\right)} .
$$

Let us now briefly discuss some properties of $M_{i}$ matrixes. Above we stated that for stable fixed points $\{x(s)\}$ all the matrixes $M_{i}$ coincide, but this is true only when $w\left(S_{0}, S_{1}, \ldots, S_{p-1}\right)$ is a symmetric function under the permutations of its arguments, which holds for MSI Hamiltonian (3). In the case when one wants to introduce also pair interactions one find that the definition 
of $M_{i}$ becomes not unique. To demonstrate this let us consider spin- $\frac{1}{2}$ Ising model on the 4-polygon (square) Bethe-like lattice with $J_{2}$ pair interactions only. In Fig. 2 one can see two configurations of spins on a 4-polygon which differ only in permutation of spins, but their statistical weights are different. For example, for configuration (1) we have

$$
\begin{aligned}
& M(+,+)=\sum_{y_{i+1}, \ldots, t_{i+1}} w\left(+, y_{i+1}, t_{i+1},+\right) x^{\gamma}\left(y_{i+1}\right) x^{\gamma}\left(t_{i+1}\right)= \\
& e^{4 \beta J_{2}}+x^{2 \gamma}+2 x^{\gamma}
\end{aligned}
$$

and for configuration (2) respectively

$$
M(+,+)=e^{4 \beta J_{2}}+e^{-4 \beta J_{2}} x^{2 \gamma}+2 x^{\gamma} .
$$

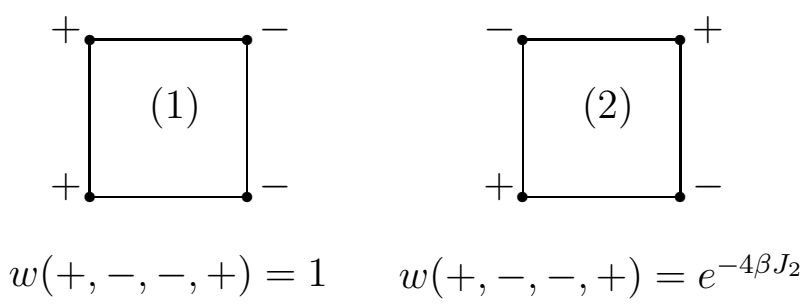

Figure 2: Two configuration of spins on a 4-polygons with asymmetric statistical weight function.

To recover the symmetry of $w$ we can add all possible diagonal interactions between the spins on a polygon (see Fig. 3). Thus, if one wants to consider pair or / and $l$-interactions $(l=2, \ldots, p-1$ is the number of interacting spins on a $p$-polygon) one has to consider all possible interactions between spins on a polygon to have a symmetric function of the statistical weight $w$ under the permutation of spins on a $p$-polygon.

In order to use the transfer-matrix method we consider only the case when $w$ is a symmetric function as described above. Now let us return to matrices $M$ and $A$. Using the symmetry property of $w$, Eq. (田) and definitions (13), (14) one can easily prove that matrices $A$ and $M$ commute. Thus they can be diagonalized simultaneously. The spectrum of matrix $A$ is highly degenerated, it has only one nonzero eigenvalue

$$
\lambda_{1}^{A}=1+\sum_{s=S-1, \ldots,-S} x^{\gamma+1}(s)
$$

with eigenvector

$$
v_{1}^{*}=\left(\lambda_{1}^{A}\right)^{-\frac{1}{2}}\left(1, x^{\frac{\gamma+1}{2}}(S-1), \ldots, x^{\frac{\gamma+1}{2}}(-S)\right) .
$$

After the diagonalization of matrix $M$ we come to the following expression for the correlation functions

$$
g(n)=\sum_{k=1}^{2 S} A_{k}\left(\frac{\lambda_{k+1}}{\lambda_{1}}\right)^{n},
$$




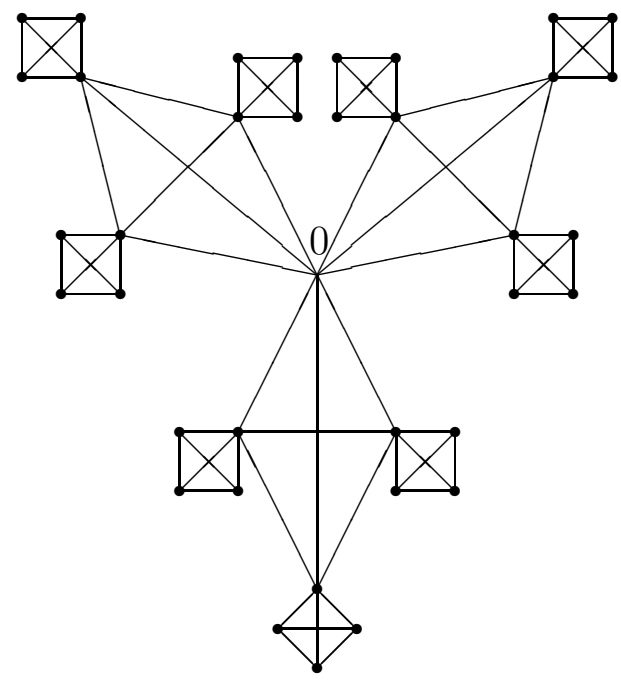

Figure 3: The schematic of the 4-polygon Bethe-like lattice with all possible pair interactions on a polygon.

where $\lambda_{k}$ are the eigenvalues of matrix $M$. The coefficients $A_{k}(k=1,2, \ldots, 2 S)$ can be obtained by solving the following system of linear algebraic equations

$$
\left\{\begin{array}{l}
g(0)=A_{1}+A_{2}+\cdots+A_{2 S}, \\
g(1)=A_{1} l_{1}+A_{2} l_{2}+\cdots+A_{2 S} l_{2 S}, \\
\vdots \\
g(2 S-1)=A_{1} l_{1}^{2 S-1}+A_{2} l_{2}^{2 S-1}+\cdots+A_{2 S} l_{2 S}^{2 S-1},
\end{array}\right.
$$

where $l_{k} \equiv \lambda_{k+1} / \lambda_{1}$. The solution of this system of equations can be presented in the form

$$
A_{k}=\frac{\sum_{j=0}^{2 S-1}(-1)^{2 S-1-j} F_{j}(k) g(j)}{\prod_{i=1}^{2 S}\left(l_{k}-l_{i}\right)}, \quad i \neq k .
$$

where $F_{j}(k)$ are the elementary functions of $2 S-1$ variables $l_{1}, l_{2}, \ldots, l_{k-1}, l_{k+1}, \ldots, l_{2 S}$ :

$$
\begin{aligned}
& F_{2 S-1}(k)=1, \\
& F_{2 S-2}(k)=l_{1}+\cdots l_{k-1}+l_{k+1}+\cdots+l_{2 S}, \\
& \vdots \\
& F_{0}(k)=l_{1} \cdots l_{k-1} l_{k+1} \cdots l_{2 S}
\end{aligned}
$$

(for more details see the Appendix of Ref.[21]). From the Eq. (18) we can find for MSI spin- $\frac{1}{2}$ Ising model $\left\langle S_{0}^{2}\right\rangle=\frac{1}{4}$ and

$$
A_{1}=g(0)=4\left\langle S_{0}^{2}\right\rangle-m_{1}^{2}=1-m_{1}^{2},
$$

for MSI spin-1 Ising model respectively

$$
A_{1}=\frac{l_{2} g(0)-g(1)}{l_{2}-l_{1}}=\frac{\lambda_{3}\left(m_{2}-m_{1}^{2}\right)-\lambda_{1}\left(\left\langle S_{0} S_{1}\right\rangle-m_{1}^{2}\right)}{\lambda_{3}-\lambda_{2}}
$$


and $A_{2}=A_{1}\left(l_{1} \Leftrightarrow l_{2}\right)$, where $m_{2}=\left\langle S_{0}^{2}\right\rangle$, etc.

Thus, Eqs. (9),(10) and (17) give us a full set of equations for investigation of spin- $S$ MSI and pair interaction models on the Bethe-like lattices.

\section{The General MSI Spin- $\frac{1}{2}$ Ising Model}

Here we apply the general technique developed in previous section to investigate the MSI spin- $\frac{1}{2}$ Ising model in an external magnetic field $h$ with Hamiltonian

$$
\mathcal{H}=-2^{p} J_{p} \sum_{<\text {polygons }>} \prod S_{p}-4 J_{2} \sum_{<i j>} S_{i} S_{j}-2 h \sum_{i} S_{i}
$$

where $S_{i}$ takes values $\pm \frac{1}{2}$, the first term describes the multisite interactions on p-polygons, the second sum goes over all possible pairs of spins on each $p$-polygon (see Sect. 2) and the third one goes over all spins on a lattice. The statistical weight of a $p$-polygon has the form

$$
w\left(S_{0}, \ldots, S_{p-1}\right)=\exp \left[2^{p} \beta J_{p} S_{0} \cdots S_{p-1}+4 \beta J_{2} \sum_{<i j>\in P} S_{i} S_{j}+\frac{2 \beta h}{q} \sum_{i \in P} S_{i}\right] .
$$

We see that $w$ is a symmetric function under the permutation of its arguments. Let us make the following notations

$$
\begin{aligned}
a_{k}= & w(\underbrace{+, \ldots,+}_{k}, \underbrace{-, \ldots,-}_{p-k}) \\
& \text { or } \\
a_{k}= & \exp \left[(-1)^{p-k} \beta J_{p}+\frac{(2 k-p)^{2}-p}{2} \beta J_{2}+\frac{(2 k-p)}{q} \beta h\right],
\end{aligned}
$$

where $k$ is the number of spins on a $p$-polygon in the state $+\frac{1}{2}, k \leq p$. Using this notations and $x_{N}(-) \equiv x_{N}$ we can present the corresponding recurrence equations (8) as follows

$$
x_{N}=f\left(x_{N-1}\right), \quad f(x)=\frac{\sum_{k=0}^{p-1} a_{k} C_{p-1}^{k} x^{(p-1-k) \gamma}}{\sum_{k=0}^{p-1} a_{k+1} C_{p-1}^{k} x^{(p-1-k) \gamma}},
$$

where $C_{p}^{k}=p ! /(k !(p-k) !)$ is the number of ways of choosing $k$ elements from $p$ ones without permutations.

The stable fixed points $x$ of the mapping (23) can be obtained from the fixed point equation

$$
x=f(x), \quad \sum_{k=0}^{p-1}\left(a_{k}-x a_{k+1}\right) C_{p-1}^{k} x^{(p-1-k) \gamma}=0
$$

and the stability condition

$$
\left|\frac{\partial}{\partial x} f(x)\right|_{x=x_{c}} \mid<1
$$

Using the identity $C_{p-1}^{k}=C_{p-2}^{k}+C_{p-2}^{k-1}$ one can rewrite the fixed point equation (24) as follows

$$
\sum_{k=0}^{p-2}\left(x^{\gamma+1} a_{k+1}-x^{\gamma} a_{k}+x a_{k+2}-a_{k+1}\right) C_{p-2}^{k} x^{(p-2-k) \gamma}=0 .
$$


In the stable fixed point the magnetization of the central site has the form

$$
m_{1}=\frac{1-x^{q}}{1+x^{q}}
$$

It is well known that a ferromagnetic system at high temperatures $\left(T>T_{c}\right)$ is in the paramagnetic phase with $m_{1}=0$ and, hence, $\mathrm{x}=1$ is a stable fixed point of this phase (see Eqs. (24)-(26)). For low temperatures $\left(T<T_{c}\right)$ the system is in the ferromagnetic phase with $m_{1} \neq 0$ and, hence, $x \neq 1$. Thus, at the critical point $\left(T=T_{c}\right)$ the fixed point $x_{c}=1$ loses its stability

$$
\left.\frac{\partial}{\partial x} f(x)\right|_{x_{c}=1}=1 \text {. }
$$

Here we demand that coefficients $a_{k}$ should be taken such that Eq.(24) has $x=1$ solution. For antiferromagnetics the critical temperature can be found from Eq. (24) and the following condition

$$
\left.\frac{\partial}{\partial x} f(x)\right|_{x=x_{c}}=-1 \text {. }
$$

From definitions (13)-(15) for stable fixed points labeling rows (columns) of the matrixes $A, A^{\prime}$ and $M$ as $+\frac{1}{2},-\frac{1}{2}$ from left to right (from up to down) we have

$$
\begin{aligned}
& M=\left(\begin{array}{ll}
\sum_{k=0}^{p-2} a_{k+2} C_{p-2}^{k} x^{(p-2-k) \gamma} & x^{\frac{\gamma-1}{2}} \sum_{k=0}^{p-2} a_{k+1} C_{p-2}^{k} x^{(p-2-k) \gamma} \\
x^{\frac{\gamma-1}{2}} \sum_{k=0}^{p-2} a_{k+1} C_{p-2}^{k} x^{(p-2-k) \gamma} & x^{\gamma-1} \sum_{k=0}^{p-2} a_{k} C_{p-2}^{k} x^{(p-2-k) \gamma}
\end{array}\right), \\
& A=\left(\begin{array}{ll}
1 & x^{\frac{\gamma+1}{2}} \\
x^{\frac{\gamma+1}{2}} & x^{\gamma+1}
\end{array}\right) \quad \text { and } \quad A^{\prime}=\left(\begin{array}{ll}
1 & -x^{\frac{\gamma+1}{2}} \\
-x^{\frac{\gamma+1}{2}} & x^{\gamma+1}
\end{array}\right) .
\end{aligned}
$$

Now from Eqs. (17) for two-spin correlation functions we can write

$$
g(n)=A_{1} \lambda^{n}
$$

where $A_{1}=1-m_{1}^{2}$ (see Eq. 19), $\lambda=\lambda_{2} / \lambda_{1}$ and $\lambda_{1}, \lambda_{2}$ are the eigenvalues of the matrix $M$

$$
\begin{aligned}
& \lambda_{1}=\sum_{k=0}^{p-2}\left(a_{k+2}+x^{\gamma} a_{k+1}\right) C_{p-2}^{k} x^{(p-2-k) \gamma}, \\
& \lambda_{2}=x^{\gamma-1} \sum_{k=0}^{p-2}\left(a_{k}-x a_{k+1}\right) C_{p-2}^{k} x^{(p-2-k) \gamma} .
\end{aligned}
$$

Let us now consider the behavior of $g(n)$ in the critical region for the ferromagnetic models. If one restricts the interactions to pair interactions $\left(J_{2}+h\right)$, the Lee-Yang circle theorem[25] establishes that only at $h=0$ a phase transition can occur. However, with multisite interactions present $(\mathrm{MSI}+h)$, phase transition may occur for $h \neq 0$ at $T_{c} \neq 0$ and for $h=0$ only at $T_{c}=0$ [8].

The $T-h$ second order phase transition region ( surface for $\left(\mathrm{MSI}+J_{2}+h\right)$, line for $(\mathrm{MSI}+h)$, and a point for $\left(J_{2}+h\right)$ interactions) can be obtained from Eq. (27)

$$
(p-1)(q-1) \sum_{k=0}^{p-2}\left(a_{k}-a_{k+1}\right) C_{p-2}^{k}=\sum_{k=0}^{p-1} a_{k+1} C_{p-1}^{k} .
$$


For example, the critical point for $\left(J_{2}+h\right)$ interaction model on the Bethe lattice $(p=2)$ is [13]

$$
\left(J_{2}\right)_{c}=\frac{1}{2} \ln \frac{q}{q-2}
$$

and for Husimi lattice $(p=3)$ is

$$
\left(J_{2}\right)_{c}=\frac{1}{4} \ln \frac{2 q+1}{2 q-3} .
$$

Let us now calculate the correlation length function $\xi$. Substituting $x_{c}=1$ into Eqs. (30)-(32) and taking into account Eq. (33) for correlation function at the critical region $g_{c}(n)$ we obtain

$$
g_{c}(n)=[(p-1)(q-1)]^{-n} .
$$

From the definition of the dimensionality presented in Sect. 1 follows that for $n \gg 1$

$$
d=\frac{n}{\ln n} \ln [(p-1)(q-1)] .
$$

Hence, $g_{c}(n)$ can be written as

$$
g_{c}(n) \sim n^{-d}
$$

Thus, near the critical region the correlation function has the form

$$
g(n)=n^{-d} \exp \left(-\frac{n}{\xi}\right)
$$

where the correlation length $\xi$ is

$$
\xi=\left[\ln \left[\frac{1}{(p-1)(q-1) \lambda}\right]\right]^{-1} .
$$

The obtained formulas (36)-(38) are in good agreement with the general behavior of correlation functions described in Ref. [13]. From Eqs. (31), (32) and (38) follows that near the critical region the correlation length $\xi$ has singularity in the form

$$
\left.\xi \sim \xi\left(J_{p}, J_{2}, h\right)\right|_{t=0} t^{-1}
$$

where

$$
\xi^{-1}\left(J_{p}, J_{2}, h\right)=\frac{\sum_{k=0}^{p-2}\left(a_{k+2}^{\prime}+a_{k+1}^{\prime}-(p-1)(q-1)\left(a_{k}^{\prime}-a_{k+1}^{\prime}\right)\right) C_{p-2}^{k}}{\sum_{k=0}^{p-2}\left(a_{k+2}+a_{k+1}\right) C_{p-2}^{k}}
$$

and $t=\frac{T-T_{c}}{T_{c}}, a_{k}^{\prime}=\left.\frac{\partial}{\partial t} a_{k}\right|_{t=0}$. Thus, the correlation length $\xi$ of ferromagnetic models on the Bethe-like lattices increases as the critical region is approached according to $\xi \sim t^{-\nu}$ with critical exponent $\nu=1$.

Further, let us calculate the bulk susceptibility per lattice site $\chi=\frac{\partial m_{1}}{\partial h}$. In our case, because we have the exact expression for correlation functions, it is easier to calculate $\chi$ using the fluctuation relation 13

$$
\chi=\left(N_{l} k T\right)^{-1} \sum_{i} \sum_{j} g_{i j},
$$


where the summation goes over all spins, $g_{i j}$ is the correlation functions of spins on sites $i, j$ and $N_{l}$ is the total number of sites on a lattice. Let us now consider the Eq. (40) in detail

$$
\chi=\frac{1}{k T} \lim _{N_{l} \rightarrow \infty} \frac{1}{N_{l}} \sum_{i} \sum_{j} \frac{1}{S^{2}}\left(\left\langle S_{i} S_{j}\right\rangle-\left\langle S_{i}\right\rangle\left\langle S_{j}\right\rangle\right) .
$$

Taking into account the translational invariance and equivalence of all sites of Bethe-like lattice (see Sect. 2) we have

$$
\begin{aligned}
\left\langle S_{i}\right\rangle & =\left\langle S_{j}\right\rangle, \\
\frac{1}{N_{l}} \sum_{i} \sum_{j}\left\langle S_{i} S_{j}\right\rangle & =\sum_{j}\left\langle S_{0} S_{j}\right\rangle .
\end{aligned}
$$

Substituting this relation into Eq. (41) we obtain

$$
\chi=\frac{1}{k T} \lim _{N_{l} \rightarrow \infty} \sum_{j} \frac{1}{S^{2}}\left(\left\langle S_{0} S_{j}\right\rangle-\left\langle S_{0}\right\rangle^{2}\right)=\beta \lim _{l \rightarrow \infty} \sum_{n=0}^{l} C_{n} g(n),
$$

where $C_{n}$ is the number of sites on the $n t h$-shell and $l$ is the number of shells on a lattice. From Eqs. (2) and (30) one can easily find

$$
\begin{aligned}
\chi= & \beta\left(1-m_{1}^{2}\right) \lim _{l \rightarrow \infty}\left[1+q(p-1) \lambda \sum_{n=1}^{l}[(p-1)(q-1) \lambda]^{n-1}\right]= \\
& \beta\left(1-m_{1}^{2}\right) \lim _{l \rightarrow \infty}\left[\frac{1+\lambda(p-1)}{1-(p-1)(q-1) \lambda}-q(p-1) \lambda \frac{[(p-1)(q-1) \lambda]^{l}}{1-(p-1)(q-1) \lambda}\right] .
\end{aligned}
$$

It is now obvious that for $(p-1)(q-1) \lambda \geq 1$ the susceptibility $\chi$ diverges and for $(p-1)(q-1) \lambda \leq$ 1 have the form

$$
\chi=\frac{\beta\left(1-m_{1}^{2}\right)(1+\lambda(p-1))}{1-(p-1)(q-1) \lambda} .
$$

From Eqs. (39) and (42) for the general ferromagnetic MSI spin- $\frac{1}{2}$ model in an external magnetic field one can easily establish the relation between the susceptibility $\chi$ and the correlation length $\xi$ in the critical region $\chi \sim \xi$.

\section{Concluding Remarks}

In this paper we have applied the general Bethe-like lattice approximation to studying the correlation functions of the most general multisite interaction (MSI) spin- $S$ Ising models in an external magnetic field. Deriving the recurrence relations and using dynamical systems theory the thermodynamic properties of the system are studied by the investigation of the behavior of the fixed points of the corresponding map. Exact formulas for the correlation functions $g(n)$ (spin- $S$ Ising models), and for the correlation length $\xi$, the bulk susceptibility

per lattice site $\chi$ (spin- $\frac{1}{2}$ Ising models) have been derived for arbitrary $h$ and $T$. A singular behavior of the correlation length $\xi$ near the critical region with critical exponent $\nu=1$ has 
been obtained exactly. We also showed that near the critical region the magnetic susceptibility $\chi$ is proportional to the correlation length $\xi$.

From Eq. (34) one can see that in the critical region the correlation functions tend to zero as $n \rightarrow \infty$ and one may try to interpret this as absence of correlation on the Bethe-like lattice models. But this is not correct, because as we can see from Eqs. (35) and (36) the fact that at $T=T_{c} g(n) \rightarrow 0$ is a consequence of infinite dimensionality of the Bethe-like lattices embedded in the Euclidean space. As we can see from Eq. (39) correlation length in fact has singularity at $T=T_{c}$. Hence, the second order phase transitions of models defined on the Bethe-like lattices have the same "attributes" as the models on regular lattices. As showed by Gujrati [12] Bethe and Bethe-like lattice approximations give qualitatively correct results even when conventional mean-field theories fail. Furthermore, due to various options regarding the connection of the basic building blocks, using Bethe-like lattices one can get better approximations with respect to mean-field and usual Bethe lattice calculations, especially in the presence of frustrations 8 .

In conclusion we want to note that the method presented here is quite general and may be used for other models also, for example the Potts model, the multilayer Ising model etc.

\section{Acknowledgements}

Author would like to thank N. S. Ananikyan and N. Sh. Izmailyan for fruitful discussions. This work was partly supported by the Grants INTAS-96-690, INTAS-97-347.

\section{References}

[1] D. F. Styer, M. K. Phani and J. L. Lebowitz, Phys.Rev. B34, 3361 (1986).

[2] M. Grimsditch, P. Louberyre and A. Polian, Phys. Rev. B33, 7192 (1986).

[3] M. Roger, J. H. Hetherington and J. M. Delrieu, Rev. Mod. Phys. 55, 1 (1983), and references therein.

[4] H. L. Scott, Phys. Rev. A37, 263 (1988).

[5] J. A. Barker, Phys. Rev. Lett. 57, 230 (1986).

[6] C. J. Thompson, Phys. Lett. A47, 23 (1974).

[7] J. R. Heringa, H. W. J. Blote and A. Hoogland, Phys. Rev. Lett. 63, 1546 (1989).

[8] J. L. Monroe, J. Stat. Phys. 65, (1991); ibid. 67, (1992); Phys. Lett. A188, 80 (1994).

[9] T. P. Eggarter, Phys. Rev. B9, 2989 (1974).

[10] C.J. Thompson, J. Stat. Phys. 27, 441 (1982).

[11] H. A. Bethe, Prog. R. Soc. London 150, 552, (1935); R. Peierls, ibid. 154, 207 (1936).

[12] P. D. Gujrati, Phys. Rev. Lett. 74, 809 (1995). 
[13] R. J. Baxter, Exactly Solved Models in Statistical Mechanics (Academic Press, London, 1981).

[14] E. Muller-Hartman, J. Zittartz, Phys. Rev. Lett. 33, 893 (1974).

[15] T. Morita, T. Horiguchi, Prog. Theoret. Phys. 54, 982 (1975).

[16] Y. K. Wang, F. Y. Wu, J. Phys. A9, 593 (1976).

[17] Z. R. Yang, C.-Y. Xu, Commun. Theoret. Phys. 22, 419 (1994).

[18] H.E. Stanley, Introduction to Phase Transitions and Critical Phenomena (Oxford University press, New York, 1971).

[19] R. B. Stinchcombe, in Correlation Functions and Quasiparticle Interactions in Condensed Matter, ed. J. Woods Halley (Plenum, New York, 1978).

[20] C. Destri et al., Phys. Lett. B278, 311 (1992).

[21] Chin-kun Hu, N. Sh. Izmailian, Phys. Rev. E58, 1 (1998).

[22] R. Mosseri and J. F. Sados, J. Phys. France Lett. 43, 1249 (1982); J. A. Miranda-Neto and Fernando Moraes, ibid. 2, 1657 (1992); ibid. 3, 29 (1993).

[23] N. S. Ananaikian, R. G. Ghulghazaryan, N. Sh. Izmailian, Int. J. Mod. Phys. B12, 2349 (1998).

[24] N. S. Ananikian, N. Sh. Izmailian, K. A. Oganessyan, Physica A254, 207 (1998); N. S. Ananikian, et al, Fractals 5, 175 (1997); N. S. Ananikian and S. K. Dallakian, Physica D107, 75 (1997).

[25] T. D. Lee and C. N. Yang, Phys. Rev. 87: 404, 410 (1952). 